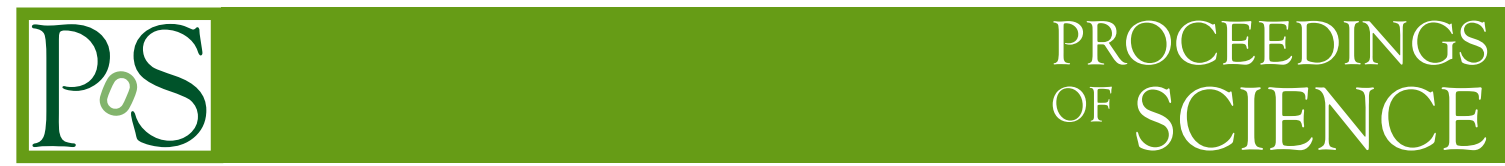

\title{
Searches for CP violation in multibody D decays
}

\author{
Jinlin Fu* $\mathbf{u}^{* \dagger}$ \\ INFN Milano \\ E-mail: j.fuecern.ch
}

Multibody charm decays are a good place to search for $C P$ violation. The results of the $\mathrm{LHCb}$ collaboration in the decays of $D^{+} \rightarrow \pi^{-} \pi^{+} \pi^{+}, D^{0} \rightarrow \pi^{+} \pi^{-} \pi^{+} \pi^{-}$and $D^{0} \rightarrow K^{+} K^{-} \pi^{+} \pi^{-}$are presented.

The 15th International Conference on B-Physics at Frontier Machines at the University of Edinburgh, $14-18$ July, 2014

University of Edinburgh, $U K$

*Speaker.

On behalf of the LHCb collaboration 


\section{Introduction}

$C P$ violation $(C P V)$ is an interference effect, in which at least two amplitudes with different strong and weak phases are required. In the standard model, $C P V$ in charm decays is very suppressed [1,2]. Multibody charm decays are a good place to search for $C P V$, due to their rich resonant structures with interfering amplitudes. In this way, $C P V$ can also be probed in different phase space regions.

In the analyses performed by the LHCb collaboration, model-independent methods are used to search for $C P V$ in 3- and 4-body decays. The measurements in the self-tagged decays of $D^{+} \rightarrow \pi^{-} \pi^{+} \pi^{+}[3]$ and $D^{*}$-tagged promptly-produced decays of $D^{0} \rightarrow \pi^{+} \pi^{-} \pi^{+} \pi^{-}$and $D^{0} \rightarrow$ $K^{+} K^{-} \pi^{+} \pi^{-}$[4] use the Miranda method. The measurements in decays of $D^{0} \rightarrow K^{+} K^{-} \pi^{+} \pi^{-}$[5] selected from semileptonic $B$ decays use the $T$-odd correlations method. Charge conjugated states are implicit, unless otherwise stated.

\section{Miranda method}

The Miranda method [6] is based on a direct comparision between the $D$ and $\bar{D}$ phase space. The multi-body phase space is divided into bins. For each bin, a significance is defined from the difference in bin occupation between $C P$ conjugate decays:

$$
S_{C P}^{i} \equiv \frac{N_{i}(D)-\alpha N_{i}(\bar{D})}{\sqrt{\alpha\left(\sigma_{i}^{2}(D)+\sigma_{i}^{2}(\bar{D})\right)}}, \alpha \equiv \frac{\sum_{i} N_{i}(D)}{\sum_{i} N_{i}(\bar{D})} .
$$

The number of signal events in each bin is given by $N_{i}$ with an uncertainty $\sigma_{i}$. The parameter $\alpha$ removes global production and detection asymmetries.

In absence of $C P V, S_{C P}$ is expected to be a Gaussian distribution with a mean of zero and a width of one. A $\chi^{2}$ statistic with $N_{\text {bins }}-1$ degree of freedom is constructed by the sum of squred $S_{C P}^{i}$,

$$
\chi^{2}=\sum_{i}\left(S_{C P}^{i}\right)^{2},
$$

from which a $p$-value is calculated. The p-value gives the probability of getting a $\chi^{2}$ as extreme as the one measured, assuming the no $C P V$ hypothesis is true.

Using $1 \mathrm{fb}^{-1}$ data sample recorded at $\sqrt{s}=7 \mathrm{TeV}$ in 2011, about 3.1 million $D^{+} \rightarrow \pi^{-} \pi^{+} \pi^{+}$ candidate decays with a purity of $82 \%$ are reconstructed. Adaptive and uniform binning schemes in the phase space are used for different binning sizes. The $\chi^{2}$ test is performed for the adaptive binning scheme, using 20,30, 40, 49 and 100 bins. All $p$-values are above 50\%, consistent with no $C P V$ hypothesis. The $\chi^{2}$ test is also performed for the uniform binning scheme, using 20, 32 , 52 and 98 bins. All $p$-values are above $90 \%$, consistent with no $C P V$ hypothesis. The results of the adaptive binning schemes with 49 and 100 bins are shown in Fig. 1.

Using the same $1 \mathrm{fb}^{-1}$ data sample, about $5.7 \times 10^{4} D^{0} \rightarrow K^{+} K^{-} \pi^{+} \pi^{-}$and $3.3 \times 10^{5} D^{0} \rightarrow$ $\pi^{+} \pi^{-} \pi^{+} \pi^{-}$decays are reconstructed from $D^{*+} \rightarrow D^{0} \pi^{+}$decays. Signal yields are extracted from a two-dimensional maximum likelihood fit in $m(h h h h)$ and $\Delta m=m\left(\pi_{s} h h h h\right)-m(h h h h)$, where $h$ represents a pion or a kaon candidate. Projection plots from fit are shown in Fig. 2. The phase space is more complicated than the one in 3-body decays, and can be described with five invariant 

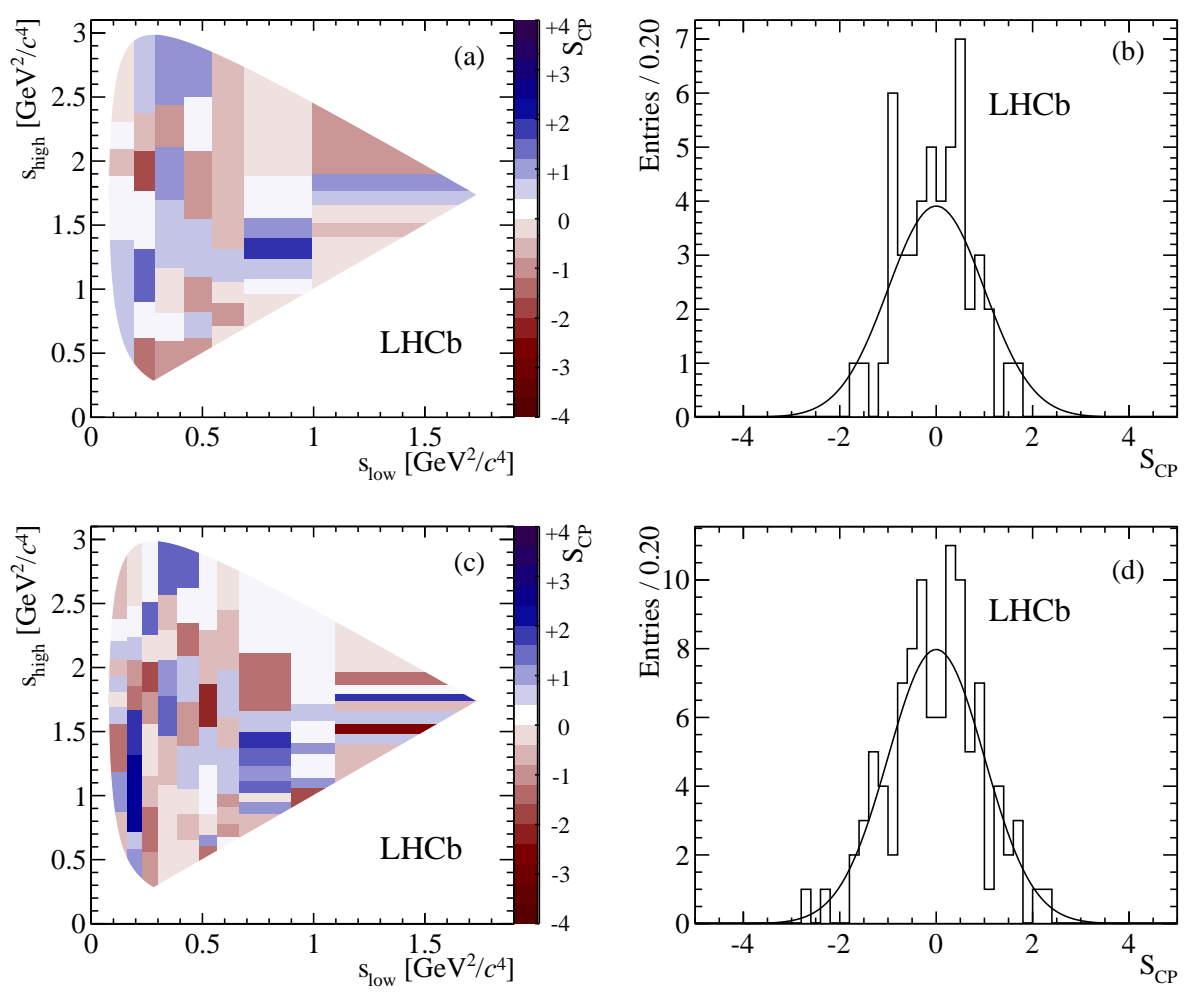

Figure 1: Distributions of $S_{C P}^{i}$ across the $D^{+}$Dalitz plane, with the adaptive binning scheme of uniform population for the total $D^{+} \rightarrow \pi^{-} \pi^{+} \pi^{+}$data sample with (a) 49 and (c) 100 bins. The corresponding onedimensional $S_{C P}^{i}$ distributions (b) and (d) are shown with a standard normal Gaussian function superimposed (solid line).

mass-squred combinations of final particles. An adaptive binning algorithm is devised to partition the phase space into 5-dimensional hypercubes. The $\chi^{2}$ test is performed using 16, 32, 64 and 256 bins. All results are consistent with no $C P V$ hypothesis. The results of 32 and 128 bins are shown in Fig. 3.

\section{3. $T$-odd correlations method}

$T$-odd correlations are defined as the triple products of final states particle momenta in the $D^{0}$ center-of-mass frame, $C_{T} \equiv \vec{p}_{K^{+}} \cdot\left(\vec{p}_{\pi^{+}} \times \vec{p}_{\pi^{-}}\right)$for $D^{0}$ and $\bar{C}_{T} \equiv \vec{p}_{K^{-}} \cdot\left(\vec{p}_{\pi^{-}} \times \vec{p}_{\pi^{+}}\right)$for $\bar{D}^{0}$ decays. Then we can build two $T$-odd observables:

$$
A_{T} \equiv \frac{\Gamma_{D^{0}}\left(C_{T}>0\right)-\Gamma_{D^{0}}\left(C_{T}<0\right)}{\Gamma_{D^{0}}\left(C_{T}>0\right)+\Gamma_{D^{0}}\left(C_{T}<0\right)}, \quad \bar{A}_{T} \equiv \frac{\Gamma_{\bar{D}^{0}}\left(-\bar{C}_{T}>0\right)-\Gamma_{\bar{D}^{0}}\left(-\bar{C}_{T}<0\right)}{\Gamma_{\bar{D}^{0}}\left(-\bar{C}_{T}>0\right)+\Gamma_{\bar{D}^{0}}\left(-\bar{C}_{T}<0\right)},
$$

where $\Gamma_{D^{0}}\left(\Gamma_{\bar{D}^{0}}\right)$ is the partial decay width of $D^{0}\left(\bar{D}^{0}\right)$ decays to $K^{+} K^{-} \pi^{+} \pi^{-}$in the indicated $C_{T}\left(\bar{C}_{T}\right)$ range. However, final state interaction (FSI) could introduce asymmetries [7, 8]. A true $C P$-violating observable is defined as:

$$
a_{C P}^{T \text {-odd }} \equiv \frac{1}{2}\left(A_{T}-\bar{A}_{T}\right)
$$



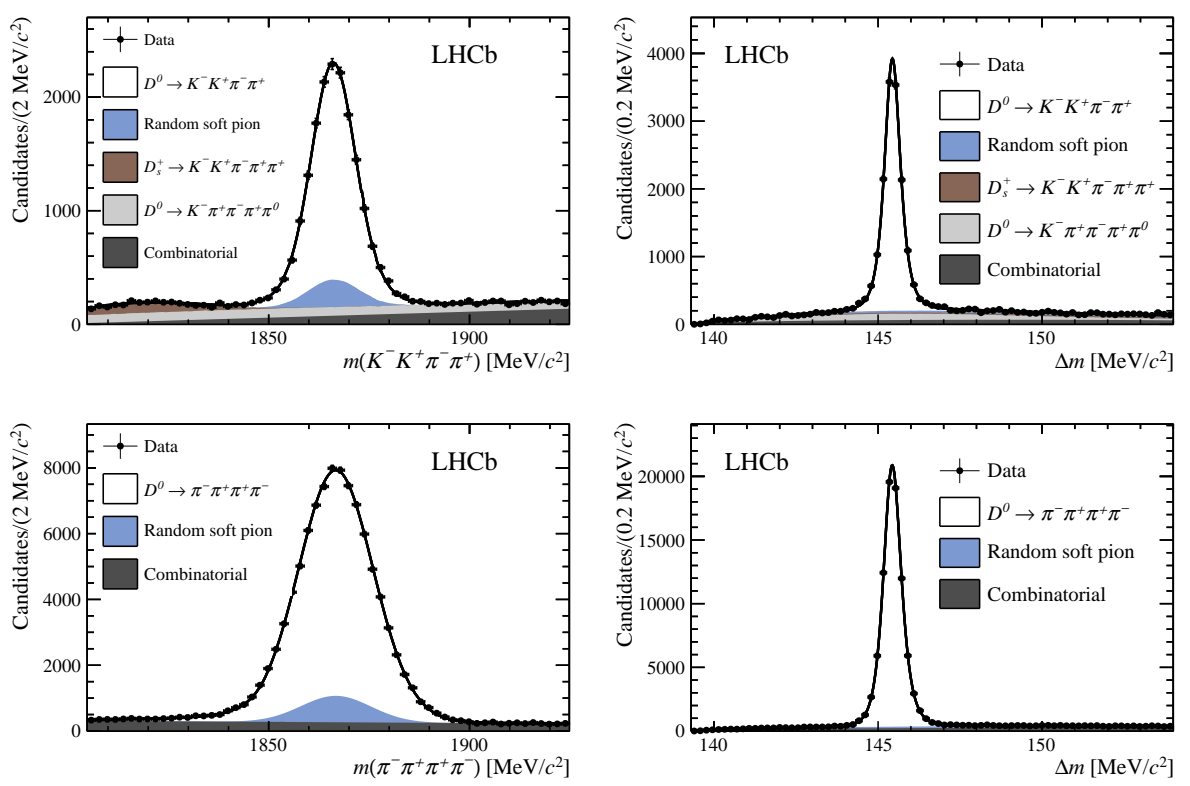

Figure 2: Distributions of (a), (c) $m(h h h h)$ and (b), (d) $\Delta(m)$ for (a), (b) $D^{0} \rightarrow \pi^{+} \pi^{-} \pi^{+} \pi^{-}$, and (c), (d) $D^{0} \rightarrow K^{+} K^{-} \pi^{+} \pi^{-}$candidates. Different backgrounds are described in [4] .

where FSI effects cancel out in the difference. The physics observables $A_{T}, \bar{A}_{T}$ and $a_{C P}^{T \text {-odd }}$ are by construction insensitive to the production asymmetry of $D^{0}$ and $\bar{D}^{0}$, detector- and reconstructioninduced charge asymmetries. In contrast to the asymmetry between the phase-space integrated rates in a $D^{0} \rightarrow V_{1} V_{2}$ decay (where $V_{i}$ indicates a vector meson), $a_{C P}^{T \text {-odd }}$ is sensitive to $C P$ violation in interference between even- and odd- partial waves of the $V_{1} V_{2}$ system [10]. Previous measurements of $a_{C P}^{T \text {-odd }}$ are compatible with no $C P V$ : FOCUS measured $a_{C P}^{T \text {-odd }}=(1.0 \pm 5.7 \pm 3.7) \%$ [11], and BaBar measured $a_{C P}^{T \text {-odd }}=(0.10 \pm 0.51 \pm 0.44) \%$ [12].

Using $1 \mathrm{fb}^{-1}$ data sample recorded at $\sqrt{s}=7 \mathrm{TeV}$ in 2011, and $2 \mathrm{fb}^{-1}$ data sample recorded at $\sqrt{s}=8 \mathrm{TeV}$ in 2012 , about $1.7 \times 10^{5} D^{0} \rightarrow K^{+} K^{-} \pi^{+} \pi^{-}$decays are reconstructed from $B \rightarrow$ $D^{0} \mu^{-} X$ decays (where $X$ indicates any system composed of charged and neutral particles). The selected data sample is split into four subsamples according to the flavor of the $D^{0}$, which is determined by the charge of the muon candidate, and the sign of $C_{T}\left(\bar{C}_{T}\right)$. A simultaneous maximum likelihood fit to the $m\left(K^{+} K^{-} \pi^{+} \pi^{-}\right)$distribution of the four subsamples is used to determine the signal number and the asymmetries $A_{T}$ and $\bar{A}_{T}$. The fit model consists of two Gaussian functions with common mean for the signal and an exponential function for the background. The two asymmetries $A_{T}$ and $\bar{A}_{T}$ are included in the fit model as

$$
\begin{aligned}
N_{D^{0}, C_{T}>0} & =\frac{1}{2} N_{D^{0}}\left(1+A_{T}\right), \\
N_{D^{0}, C_{T}<0} & =\frac{1}{2} N_{D^{0}}\left(1-A_{T}\right), \\
N_{\bar{D}^{0},-\bar{C}_{T}>0} & =\frac{1}{2} N_{\bar{D}^{0}}\left(1+\bar{A}_{T}\right), \\
N_{\bar{D}^{0},-\bar{C}_{T}<0} & =\frac{1}{2} N_{\bar{D}^{0}}\left(1-\bar{A}_{T}\right) .
\end{aligned}
$$



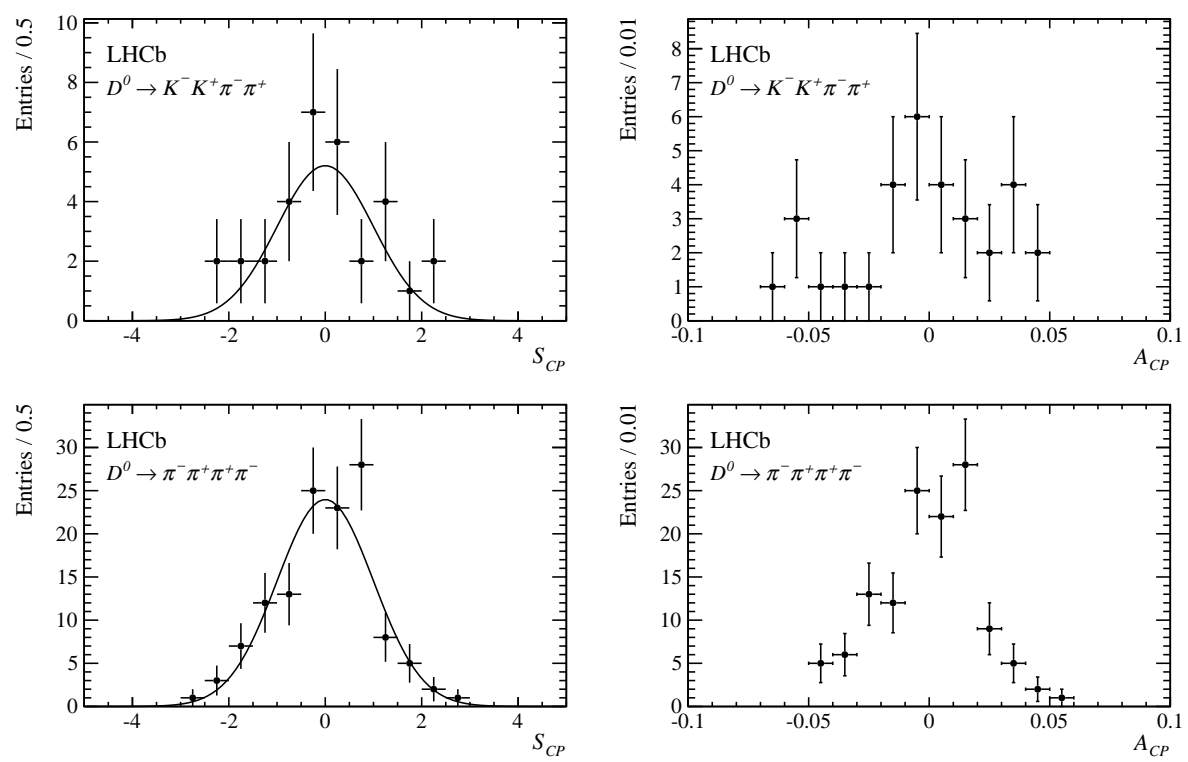

Figure 3: Distributions of (a), (c) $S_{C P}$ and (b), (d) local CP asymmetry per bin for (a), (b) $D^{0} \rightarrow K^{+} K^{-} \pi^{+} \pi^{-}$ decays with 32 bins, for (c), (d) $D^{0} \rightarrow \pi^{+} \pi^{-} \pi^{+} \pi^{-}$decays with 128 bins.
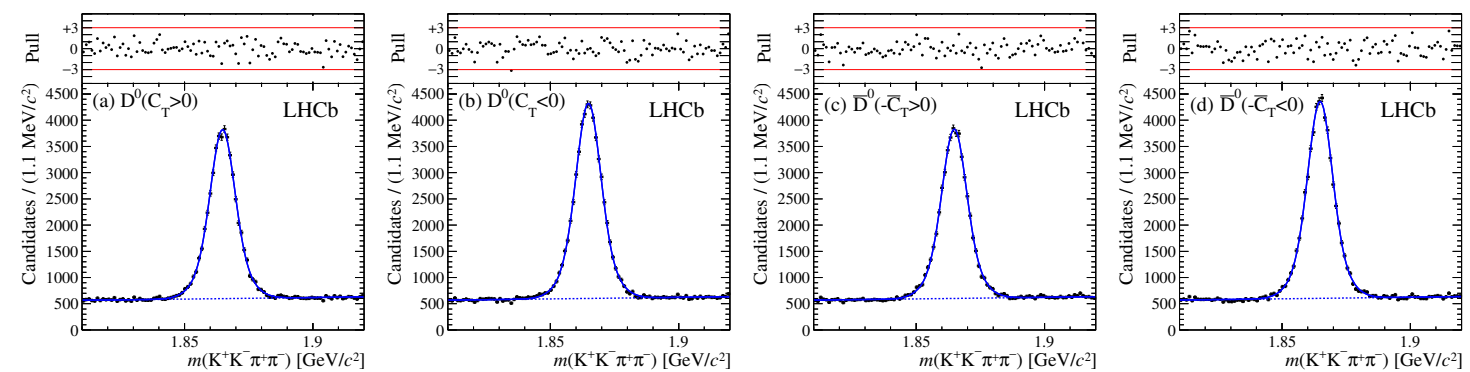

Figure 4: Distributions of the $K^{+} K^{-} \pi^{+} \pi^{-}$invariant mass in the four samples defined by $D^{0}\left(\bar{D}^{0}\right)$ flavour and the sign of $C_{T}\left(\bar{C}_{T}\right)$. The results of the fit are overlaid as a solid line, and a dashed line is used for representing the background. The normalised residuals (pulls) of the difference between the fit results and the data points, divided by their uncertainties, are shown on top of each distribution.

The $C P$-violating asymmetry $a_{C P}^{T \text {-odd }}$ is then calculated from $A_{T}$ and $\bar{A}_{T}$. The results of the fit are shown in Fig. 4.

Three different approaches have been followed to search for $C P V$ : the phase space integrated measurement, measurements in different regions of phase space, and measurements as a function of the $D^{0}$ decay time. By fitting the full data sample, the results of the first approach are:

$$
\begin{aligned}
A_{T} & =(-7.18 \pm 0.41 \text { (stat) } \pm 0.13(\text { syst })) \%, \\
\bar{A}_{T} & =(-7.55 \pm 0.41 \text { (stat) } \pm 0.12(\text { syst })) \%, \\
a_{C P}^{T \text {-odd }} & =(0.18 \pm 0.29(\text { stat }) \pm 0.04(\text { syst })) \%,
\end{aligned}
$$

The relatively large asymmetries observed in $A_{T}$ and $\bar{A}_{T}$ are due to FSI effects [7, 8], which are difficult to predict [9]. $a_{C P}^{T \text {-odd }}$ is consistent with no $C P V$ hypothesis. 

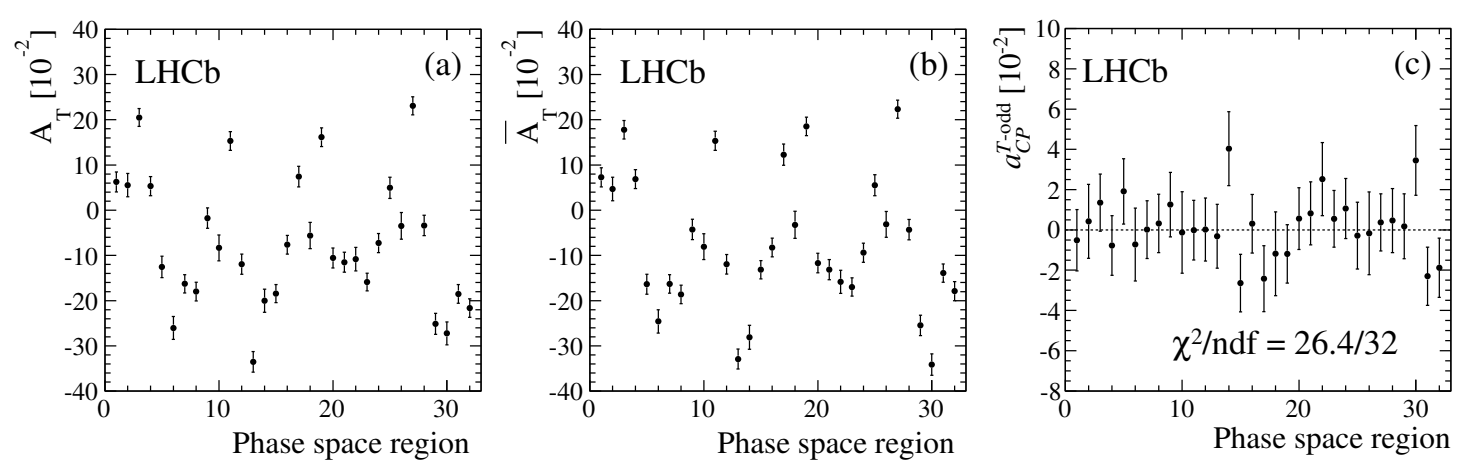

Figure 5: Distributions of the asymmetry parameters (a) $A_{T}$, (b) $\bar{A}_{T}$ and (c) $a_{C P}^{T \text {-odd }}$ in 32 regions of the phase space.

The phase space is divided into 32 regions by choosing the Cabibbo-Maksimowicz [13] variables as basis for the binning scheme. The compatibility with the CP conservation hypothesis is tested by means of a $\chi^{2}$ test,

$$
\chi^{2}=R^{T} V^{-1} R
$$

where $R$ is the array of $a_{C P}^{T \text {-odd }}$ measurements, and $V^{-1}$ is the inverse of the covariance matrix $V$, defined as the sum of the statistical and systematic covariance matrices. The distributions of the asymmetries are shown in Fig. 5. The distribution of $a_{C P}^{T \text {-odd }}$ results is consistent with no $C P V$ hypothesis with a $p$-value of $74 \%$. The $A_{T}$ and $\bar{A}_{T}$ are significantly different among the different regions, which can be explained by the rich resonant structure of the hadronic four-body decay [14] that produces different FSI effects over the phase space. Four alternative binning schemes, one with 8 regions and three with 16 regions, are also tested. All $p$-value are above $20 \%$, consistent with no $C P V$ hypothesis.

The $D^{0}$ decay time is divided into 4 regions. The asymmetry distributions are shown in Fig. 6. The $a_{C P}^{T \text {-odd }}$ results are consistent with no $C P V$ hypothesis with a $p$-value of $86 \%$. The $A_{T}$ and $\bar{A}_{T}$ do not show any significant dependence as a function of the decay time, and are compatible with constant functions with $p$-values above $30 \%$. Consistent results are obtained when using 3 or 5 regions of $D^{0}$ decay time in agreement with no $C P V$ hypothesis with $p$-values above $80 \%$.

\section{Summary}

Searches for $C P V$ in 3- and 4-body D decays are performed by the LHCb experiment using data collected from $p-p$ collisions at $\sqrt{s}=7 \mathrm{TeV}\left(1 \mathrm{fb}^{-1}\right)$ and $8 \mathrm{TeV}\left(2 \mathrm{fb}^{-1}\right)$. From the $1 \mathrm{fb}^{-1}$ dataset, a search for $C P V$ using the Miranda method is performed in $D^{+} \rightarrow \pi^{-} \pi^{+} \pi^{+}$, prompt $D^{0} \rightarrow \pi^{+} \pi^{-} \pi^{+} \pi^{-}$and $D^{0} \rightarrow K^{+} K^{-} \pi^{+} \pi^{-}$decays. A search for $C P V$ using the $T$-odd method is performed in $D^{0} \rightarrow K^{+} K^{-} \pi^{+} \pi^{-}$decays, with the whole $3 \mathrm{fb}^{-1}$ dataset, using semileptonic tagged decays, in which three approaches are exploited: a measurement integrated over the phase space, measurements in different regions of the phase space, and measurements as a function of the $D^{0}$ decay time. These results are consistent with no $C P V$ hypothesis. 

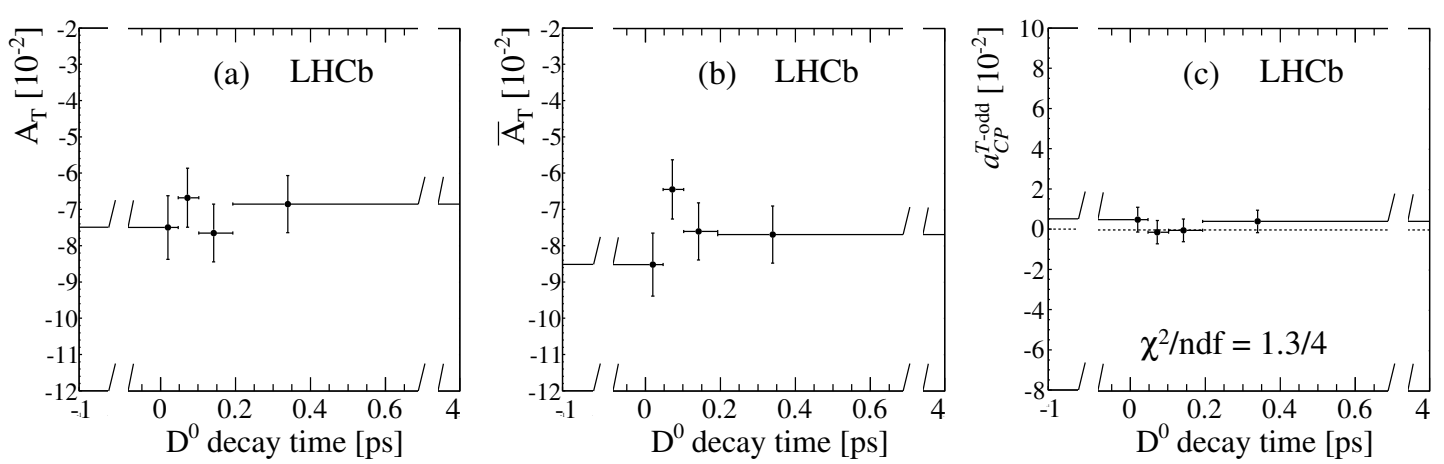

Figure 6: Distributions of the asymmetry parameters (a) $A_{T}$, (b) $\bar{A}_{T}$ and (c) $a_{C P}^{T \text {-odd }}$ as a function of the $D^{0}$ decay time. For $a_{C P}^{T \text {-odd }}$, the value of the $\chi^{2} /$ ndf for the $C P$ conservation hypothesis, represented by a dashed line, is also quoted. The scale is broken for the first and last bin.

\section{References}

[1] S. Bianco, F. L. Fabbri, D. Benson, and I. Bigi, A Cicerone for the physics of charm, Riv. Nuovo Cim. 26N7 (2003) 1, arXiv:hep-ex/0309021.

[2] Y. Grossman, A. L. Kagan, and Y. Nir, New physics and CP violation in singly Cabibbo suppressed D decays, Phys. Rev. D75 (2007) 036008, arXiv:hep-ph/0609178.

[3] R. Aaij et al. [LHCb Collaboration], Phys. Lett. B728 (2014) 585, arXiv:hep-ph/1310.7953.

[4] R. Aaij et al. [LHCb Collaboration], Phys. Lett. B726 (2013) 623, arXiv:hep-ph/1308.3189.

[5] R. Aaij et al. [LHCb Collaboration], JHEP 10 (2014) 005, arXiv:hep-ph/1408.1299.

[6] I. Bediaga et al., "On a CP anisotropy measurement in the Dalitz plot", Phys. Rev. D80, 096006 (2009).

[7] I. Bigi, Charm physics: like Botticelli in the Sistine Chapel, arXiv:hep-ph/0107102, Invited talk given at KAON 2001, the 3th International Conference on CP Violation, Pisa, Italy, June 12 - 17, 2001.

[8] M. Gronau and J. L. Rosner, Triple-product asymmetries in K, D(s), and B(s) decays, Phys. Rev. D84 (2011) 096013, arXiv:1107.1232.

[9] M. Gronau, Resonant two-body D decays, Phys. Rev. Lett. 83 (1999) 4005, arXiv:hep-ph/9908237.

[10] G. Valencia, Angular correlations in the decay $B \rightarrow V V$ and $C P$ violation, Phys. Rev. D39 (1989) 3339.

[11] FOCUS collaboration, J. M. Link et al., Search for $T$ violation in charm meson decays, Phys. Lett. B622 (2005) 239, arXiv:hep-ex/0506012.

[12] BaBar collaboration, P. del Amo Sanchez et al., Search for $C P$ violation using $T$-odd correlations in $D^{0} \rightarrow K^{+} K^{-} \pi^{+} \pi^{-}$decays, Phys. Rev. D81 (2010) 111103, arXiv:1003.3397.

[13] N. Cabibbo and A. Maksymowicz, Angular correlations in Ke-4 decays and determination of low-energy $\pi$ - $\pi$ phase shifts, Phys. Rev. 137 (1965) B438.

[14] CLEO collaboration, M. Artuso et al., Amplitude analysis of $D^{0} \rightarrow K^{+} K^{-} \pi^{+} \pi^{-}$, Phys. Rev. D85 (2012) 122002, arXiv:1201.5716. 